\title{
Pharmacokinetic-pharmacodynamic target attainment and clinical outcomes in patients treated with oral flucloxacillin plus probenecid
}

\author{
Philip Drennan ${ }^{1}$, Jared Green ${ }^{2}$, Sharon Gardiner ${ }^{2}$, Sarah Metcalf ${ }^{2}$, Carl Kirkpatrick ${ }^{3}$, \\ Richard Everts ${ }^{4}$, Mei Zhang ${ }^{5}$, and Stephen Chambers ${ }^{6}$ \\ ${ }^{1}$ Royal Prince Albert Hospital \\ ${ }^{2}$ Christchurch Hospital \\ ${ }^{3}$ Monash University \\ ${ }^{4}$ Nelson Bays Primary Health \\ ${ }^{5}$ University of Otago \\ ${ }^{6}$ University of Otago Christchurch
}

December 28, 2020

\begin{abstract}
Aim Oral flucloxacillin may be co-administered with probenecid to increase flucloxacillin concentrations and increase attainment of pharmacokinetic-pharmacodynamic (PK-PD) targets. The aims of this study were to describe outcomes of patients treated with oral flucloxacillin plus probenecid as follow-on therapy from initial intravenous treatment, and to identify optimal dosing regimens when treating infections caused by susceptible Gram-positive organisms. Methods We performed a prospective observational study of adults treated with oral flucloxacillin $1000 \mathrm{mg}$ and probenecid $500 \mathrm{mg}$ 8-hourly (with food) for proven or probable staphylococcal infections. We developed a population pharmacokinetic model of free flucloxacillin concentrations within Monolix, in order to estimate probability of PK-PD target attainment (fT $>$ MIC), and used Monte Carlo simulation to explore optimal dosing regimens. Results The 45 patients (73\% male) had a median (range) age of 49 years (20 - 74), weight of $90 \mathrm{~kg}(59-167)$, fat free mass (Janmahasatian) of $65 \mathrm{~kg}(38-89)$ and eGFR (CKD-EPI) of $89 \mathrm{~mL} / \mathrm{min} / 1.73 \mathrm{~m} 2(41-124)$. The most common infections were osteomyelitis $(n=18,40 \%)$ and septic arthritis $(n=12,27 \%)$. Forty patients $(89 \%)$ were cured 30 days after completion of therapy. $10(22 \%)$ experienced nausea which did not require treatment alternation. Free flucloxacillin clearance depended on allometrically-scaled fat free mass, and increased by $1 \%$ for each unit increase in eGFR. Conclusion Oral flucloxacillin and probenecid was well-tolerated and efficacious. Patients with higher fat free mass and eGFR may require four times daily dosing and/or therapeutic drug monitoring to ensure PK-PD target attainment.
\end{abstract}

\section{Hosted file}

fluclox_probenecid_main_submission_BJCP.pdf available at https://authorea.com/users/385854/ articles/501083-pharmacokinetic-pharmacodynamic-target-attainment-and-clinical-outcomesin-patients-treated-with-oral-flucloxacillin-plus-probenecid 